\title{
The bias flow nitrogen washout technique for measuring the functional residual capacity in infants
}

\author{
M.G. Morris*, P. Gustafsson\#, R. Tepper ${ }^{\varpi}$, M. Gappa ${ }^{+}$, J. Stocks ${ }^{\S}$, on behalf of the ERS/ATS Task \\ Force on Standards for Infant Respiratory Function Testing
}

The bias flow nitrogen washout technique for measuring the functional residual capacity in infants. M.G. Morris, P. Gustafsson, R. Tepper, M. Gappa, J. Stocks, on behalf of the ERS/ATS Task Force on Standards for Infant Respiratory Function Testing. (C) ERS Journals Ltd 2001.

ABSTRACT: The functional residual capacity (FRC) is the most commonly measured static lung volume in infants. It is important for interpreting volume-dependent pulmonary mechanics, e.g. airway resistance, and defining normal lung growth. The bias flow nitrogen washout technique is widely used for measuring FRC because the dead space and circuit resistance are low, making it suitable for small or sick infants. Moreover, data acquisition and calculation are easily programmed for a personal computer.

The aim of this paper is to provide recommendations pertaining to equipment requirements, study procedures and reporting of data for functional residual capacity measurements. While measuring the functional residual capacity is regarded as physiologically and clinically important, the accuracy of the measurement is undoubtedly equally important. Hence, the paper also emphasizes factors influencing the accuracy of functional residual capacity measurements independent of equipment requirements. These recommendations represent the "State of the Art" in 2000. Eur Respir J 2001; 17: 529-536.
*Section of Pediatric Pulmonary Medicine, University of Arkansas for Medical Sciences Little Rock, AR, USA, ${ }^{\#}$ Dept of Paediatrics, Central Hospital Skövde, Sweden, "Section of Pediatric Pulmonology, Indiana University Medical Centre, James Whitcomb Riley Memorial Hospital for Children Indianapolis, IN, USA, ${ }^{+}$University Children's Hospital, Dept of Paediatric Pulmonology and Neonatology, Medizinische Hochschule Hannover, D-30623 Hannover, Germany and ${ }^{8}$ Portex Anaesthesia, Intensive Therapy and Respiratory Medicine Unit, Institute of Child Health London, UK.

Correspondence: M.G. Morris, University of Arkansas for Medical Sciences, Section of Pediatric Pulmonary Medicine, Arkansas Children's Hospital, 800 Marshall Street Little Rock, AR 72202, USA. Fax: 15013203930

Keywords: Functional residual capacity, gas dilution, infant, lung volume, nitrogen washout, respiratory function tests

Received: August 172000

Accepted after revision October 15 2000

This work was supported by a grant from the European Respiratory Society, and by donations from Glaxo-Wellcome (UK) and GlaxoWellcome AB (Sweden). M.G. Morris was supported by a Clinical Research Grant (CG-008-N) co-funded by the American Lung Association (ALA) and the Arkansas Chapter of ALA

Previous articles in this series: No.1: U. Frey, J. Stocks, A. Coates, P.D. Sly, J. Bates, on behalf of the ERS/ATS Task Force on Standards for Infant Respiratory Function Testing. Specifications for equipment used for infants pulmonary function testing. Eur Respir J 2000; 16: 731 - 740. No. 2: P.D. Sly, R. Tepper, M. Henschen, M. Gappa, J. Stocks, on behalf of the ERS/ATS Task Force on Standards for Infant Respiratory Function Testing. Tidal Forced Expirations. Eur Respir J 2000; 16: 741 - 748 No. 3: U. Frey, J. Stocks, P. Sly, J. Bates, on behalf of the ERS/ATS Task Force on Standards for Infant Respiratory Function Testing. Specifications for signal processing and data handling used for infant pulmonary function testing. Eur Respir J 2000; 16: 1016-1022. No. 4: J.H.T. Bates, G. Schmalisch, D. Filbrun, J. Stocks, on behalf of the ERS/ATS Task Force on Standards for Infant Respiratory Function Testing. Tidal breath analysis for infant pulmonary function testing. Eur Respir J 2000; 16: 1180-1192. No.5: M. Gappa, A.A. Colin, I. Goetz, J. Stocks, on behalf of the ERS/ATS Task Force on Standards for Infant Respiratory Function Testing. Passive respiratory mechanics: The occlusion techniques. Eur Respir J 2001; 17: 141 148. No. 6: J. Stocks, S. Godfrey, C. Beardsmore, E. Bar-Yishay, R. Castile, on behalf of the ERS/ATS Task Force on Standards for Infant Respiratory Function Testing. Plethysmographic measurements of lung volume and airway resistance. Eur Repir J 2001; 17: 302-31. 
The present paper represents the consensus of investigators from the American Thoracic Society/ European Respiratory Society Task Force on Standards for Infant Lung Function tests, who have considerable experience in the measurement of lung volume in infants. The recommendations contained within deal with equipment requirements, study procedures and reporting of data for measurements of the functional residual capacity (FRC) by nitrogen $\left(\mathrm{N}_{2}\right)$ washout. It is anticipated that as knowledge progresses these guidelines will be updated regularly. Furthermore, the acceptance and application of these recommendations will be of particular value when attempting to compare data between centres, develop or use reference data, or participate in multicentre trials which use FRC as an outcome measure.

FRC has been the only static lung volume routinely measured in infants [1, 2]. FRC is important for interpreting volume-dependent pulmonary mechanics such as airway resistance or forced expiratory flows, and for defining normal lung growth. Longitudinal measurements of FRC are useful in infants with suspected impairment of alveolar growth such as in premature infants or pulmonary hypoplasia. Atelectasis, decreased lung or increased chest compliance also decreases FRC. Increased FRC commonly occurs in relation to gas trapping associated with airway obstruction. Measuring FRC has been clinically useful in the evaluation of infants with lung diseases, such as bronchiolitis, bronchopulmonary dysplasia, cystic fibrosis, and respiratory distress syndrome, as well as the evaluation of treatment efficacy [1-3].

FRC can be measured by body plethysmography (FRCpleth) or by multiple breath inert gas (x) washout/ dilution technique (FRCgasx). The latter includes the closed circuit (e.g. closed circuit helium dilution) and the open washout systems. The latter in turn can be divided into bias flow and breath-by-breath washout systems. Different inert gas tracers can be used such as $\mathrm{N}_{2}$, helium (He), argon (Ar), and sulphur hexafluoride (SF6). The gases can be measured with various devices such as a helium catharometer, $\mathrm{N}_{2}$ emission spectrophotometer (known as the $\mathrm{N}_{2}$ analyser), mass spectrometer, main stream or side stream infrared (JR) detector. Breath-by-breath systems provide information on gas distribution and ventilatory efficiency that are not provided by the bias flow washout systems. $\mathrm{N}_{2}$ washout employs $100 \%$ oxygen $\left(\mathrm{O}_{2}\right)$, but room air can be used when other inert gas tracers are washed out. $\mathrm{N}_{2}$ washout can also be performed using $21 \% \mathrm{O}_{2} / 79 \% \mathrm{Ar}$ mixture when using a mass spectrometer. The bias flow $\mathrm{N}_{2}$ washout technique has been the most commonly used approach. It is relatively simple and inexpensive to set up in the laboratory [1-14].

\section{Definitions and terminology}

The technique referred to in the present paper is for measuring the FRC in spontaneously breathing infants by the bias flow $\mathrm{N}_{2}$ washout technique. The FRC is the volume of air contained in the lung and airways at endtidal expiration [1-3]. The FRC measured is referred to as $\mathrm{FRCN}_{2}$ to differentiate it from measurements made with other tracer gases or plethysmography. For measurements of $\mathrm{FRCN}_{2}$ in ventilated infants the reader is referred to recent publications $[6,10,15-19]$.

\section{Equipment}

See previous publications in this series [20, 21] for further details and justification of the recommendations presented.

\section{The nitrogen washout circuit}

Further details of the theoretical background and basic methodology have been described by TEPPER et al. [3].

Concept. The open circuit $\mathrm{N}_{2}$ washout method for assessment of $\mathrm{FRCN}_{2}$ entails measuring the volume of nitrogen expired after end-tidal expiratory switching of the inspired gas from room air to $100 \% \mathrm{O}_{2}$. At a constant bias flow that exceeds the infant's inspiratory peak flow during tidal breathing, the integrated mixed expired $\mathrm{FN}_{2}$ (area under the curve of the $\mathrm{N}_{2}$ concentration $(\mathrm{F}=$ fractional concentration) versus time $(t))$ is multiplied by the constant flow of $\mathrm{O}_{2}\left(V^{\prime}\right)$, to obtain the volume of expired $\mathrm{N}_{2}\left(V \mathrm{~N}_{2}\right)[3,5,6]$ :

$$
V \mathrm{~N}_{2}(t)=V^{\prime} \int_{0}^{t} \mathrm{FN}_{2}(t) d t
$$

Therefore, the accuracy of the method depends on two conditions: 1) the background of $\mathrm{O}_{2}$ flow remaining constant; and 2) the exhaled $\mathrm{N}_{2}$ being well mixed with the bias flow of $\mathrm{O}_{2}$ before the $\mathrm{N}_{2}$ concentration is analysed [5].

A two-point calibration is performed with known air volumes. With the amount of $\mathrm{N}_{2}$ washed out measured and the initial fractional alveolar $\mathrm{N}_{2}$ concentration $\left(\mathrm{FAi}, \mathrm{N}_{2}\right)$ known $\left(\mathrm{FAi}_{2} \mathrm{~N}_{2}\right.$ : room air $\left.=0.79\right)$, then the lung volume at which the washout was initiated can be calculated:

$$
\text { Lung volume }(\mathrm{FRC})=\frac{\text { Volume } \mathrm{N}_{2} \text { washed out }}{\left(\mathrm{FAi}, \mathrm{N}_{2}-0.02\right)}
$$

The value of 0.02 is subtracted from $\mathrm{FAi}_{1} \mathrm{~N}_{2}$ because the washout is terminated at an end-tidal $\mathrm{FN}_{2}$ of 0.02 at the airway opening. Since the $\mathrm{N}_{2}$ is diluted within a mixing chamber (see later), the washout is actually continued until the nitrogen analyser reads an $\mathrm{FN}_{2}$ of $\leqslant 0.0065$ in the chamber, which corresponds to the end-tidal $\mathrm{FN}_{2}$ of 0.02 . This dilution effect will depend upon the flow of gas through the $\mathrm{N}_{2}$ mixing chamber and the size of the chamber. The end-tidal $\mathrm{FN}_{2}$ cut-off of 0.02 reduces the overestimation of FRC, since previous tissue $\mathrm{N}_{2}$ elimination studies during pure $\mathrm{O}_{2}$ breathing in adults and animals suggested that $\mathrm{FRCN}_{2}$ includes tissue- $\mathrm{N}_{2}$ dissolved into the lung during prolonged washout [4, 22-24].

Corrections are made for the dead space of the mask and apparatus, the switching error above FRC and 
body temperature, pressure and saturation (BTPS) (see later).

\section{Equipment required}

The equipment required is outlined in fig. 1 and includes a: clear face mask; three-way switching valve with two inlet/outlet ports and a mask port; T-piece; pneumotachometer (PNT); $\mathrm{O}_{2}$ supply with a precision flowmeter $\left(0-15 \mathrm{~L} \cdot \mathrm{min}^{-1}\right) ; \mathrm{N}_{2}$ analyser and mixing chamber; calibrating syringe; collapsible breathing bag and a T-connection (recommended; see later) [11, 12].

Face mask. The face mask and the estimation of the effective dead space have been described in detail elsewhere $[20,25]$.

Three-way switching valve. The three-way switching valve allows the infant to breathe either room air through the PNT or $100 \% \mathrm{O}_{2}$ through a T-piece. Computer-controlled electronic switching of the threeway valve via a computer keyboard stroke is preferred. The PNT is connected to one of the two-inlet/outlet ports of the three-way valve (fig. 1). The other inlet/ outlet port is attached to the T-piece which carries the constant $\mathrm{O}_{2}$ bias flow from the flowmeter, through the T-piece, tubing and $\mathrm{N}_{2}$ mixing chamber. The infant breathes room air through the face mask, mask port of the three-way valve and PNT. When switched into pure $\mathrm{O}_{2}$ during the washout, the infant no longer breathes through the PNT (fig. 1) [7, 12]. Connecting the PNT to the inlet/outlet port of the three-way valve is the preferred placement because this decreases the dead space and resistance during the washout. The PNT may be placed within the washout circuit by connecting it to the mask port, which is useful in preterm infants with unstable breathing patterns. However, variations in gas temperature, composition and viscosity need to be accounted for as well as the phase shift between the flow and $\mathrm{N}_{2}$ concentration signals [3].

The operator should be able to choose one of two switching modes: 1) automatic, whereby the software programme monitors stability of the tidal volume $(V \mathrm{~T})$ and expiatory time $(t \mathrm{E})$ so that when the user triggers the activation of the slide valve, it occurs at the end of the next expiration provided that $V \mathrm{~T}$ and $t \mathrm{E}$ of that breath are within a set percentage (e.g. 10\%) of the previous mean of $5-10$ breaths. This may be difficult to achieve in small babies with rapid or irregular breathing, and it may therefore, be necessary to allow user adjustment of the selected percentage. Nevertheless, this mode has the advantage of being simple to use and minimizing inter-observer variability. For a detailed discussion regarding the use of automatic breath identification, see BATES et al. [26]. 2) Manual, whereby the operator waits until a stable tidal breathing (end-expiratory level) pattern is observed in real time on the computer monitor and activates the slide valve as close as possible to end-expiration in a chosen breath [3, 7, 12]. This mode requires an experienced operator but is useful if the infant has irregular breathing.

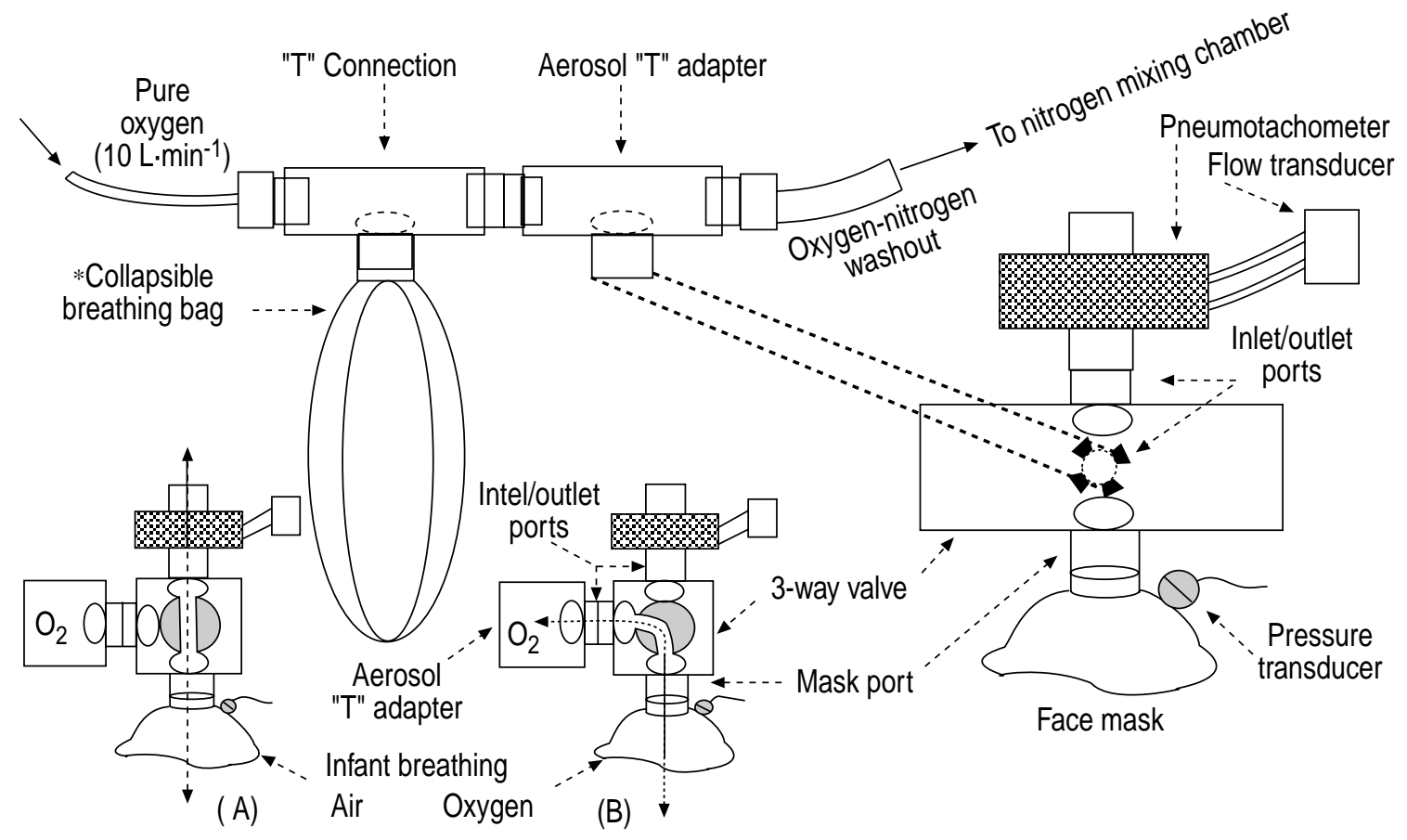

Fig. 1. - The nitrogen washout circuit. The long parallel dotted lines point to the connection site of the central port of the aerosol "T" adapter which carries the constant $\left(10 \mathrm{~L} \cdot \mathrm{min}^{-1}\right)$ bias flow of oxygen into the inlet/outlet port of the slide valve. Side views of the circuit depict the path (arrows interconnected by a dotted line) of air (A) and, after activating the slide valve, of oxygen (B) in and out of the of the infant's airway. Note that after the slide valve switches the infant into oxygen, the infant no longer breathes through the pneumotachometer. *: Inclusion of a collapsible breathing bag is optional. (Adapted and modifed from Morris [12]). 
Nitrogen analyser. $\mathrm{N}_{2}$ concentration is usually measured using emission spectrophotometry in a lowpressure ionization chamber under conditions of constant flow, although a mass spectrometer can also be used. An adjustable needle valve mounted on the mixing chamber is connected to a vacuum pump in order to provide the optimum negative pressure and constant flow for the ionization of nitrogen. The analyser should have: a linear output with a range $0-100 \% \mathrm{~N}_{2}$; an accuracy of $1 \%$ full range; a resolution of $0.01 \%$; a drift $<0.2 \% \mathrm{~N}_{2} \cdot \mathrm{h}^{-1}$ (stability close to zero $\mathrm{N}_{2}$ concentration is particularly important); a range of gas sampling rate $9-50 \mathrm{~mL} \cdot \mathrm{min}^{-1}$; a recorder output of $0-10$ Volts Direct Current (VDC) full scale; a response time $(10-90 \%$ full scale) of $<100 \mathrm{~ms}$.

The manufacturer should provide details regarding the necessary warming time for the equipment [11].

It is anticipated that these requirements will need to be amended for use with newer generations of equipment or alternative ways of measuring $\mathrm{FRCN}_{2}$, such as those based on $\mathrm{CO}_{2}$ and $\mathrm{O}_{2}$ subtraction techniques, which have not yet been validated or sufficiently tried in infant testing. Time characteristics of these systems may have to be assessed using Fast Fourier Transform (FFT) analysis of the $\mathrm{N}_{2}$ signal using a rapid $\mathrm{N}_{2}$ analyser.

Nitrogen mixing chamber. The mixing chamber should contain at least three serial channels with baffles to recirculate and mix the gas prior to exiting the chamber. A long $(\sim 2.0 \mathrm{~m})$, low resistance tubing is attached to the outlet port of the mixing chamber to prevent ambient air from diffusing back into the mixing chamber. A chamber volume of about $500 \mathrm{~mL}$ would be adequate, even in toddlers, particularly if a collapsible breathing bag is incorporated in the circuit (see later) [11, 12].

Collapsible breathing bag. A collapsible breathing bag $(0.5 \mathrm{~L})$ can be incorporated into the washout circuit via a second T-connection. It should be placed between the infant and the $\mathrm{O}_{2}$ source but closer to the former. This has been reported to enhance the reproducibility of measurements by acting as a buffer reservoir. This minimizes flow swings within the $\mathrm{N}_{2}$ mixing chamber during the breathing cycle as well as the retrograde movement of mixed $\mathrm{O}_{2}$ and $\mathrm{N}_{2}$ gas after it passes beyond the $\mathrm{N}_{2}$ sampling needle port. In addition, the bag can be used to monitor the infant's breathing pattern both during the washout and when determining the "end of test" (see later) [11, 12].

Bias flow of oxygen. The bias flow of $\mathrm{O}_{2}$ should be standardized to facilitate comparison of washout times between laboratories: $10 \mathrm{~L} \cdot \mathrm{min}^{-1}$ and $5 \mathrm{~L} \cdot \mathrm{min}^{-1}$ are suitable for infants weighing $\geqslant 5.0 \mathrm{~kg}$, respectively [3, 12]. Nevertheless, the volume of the washout circuit may influence the circuit time constant.

Flowmeter. This has been described elsewhere [20, 26, 27].

Calibration syringe. The exact dead space of the calibration syringe should be known. The combined volume of the syringe once connected to the mask port is generally less than the sum of their individual volumes as determined by water replacement. If both have been made by the same manufacturer, the latter can provide these data, but further confirmation by the investigator using water displacement with the equipment assembled as for use in vivo is recommended [11].

Measurements in infants who require an oxygen supplement. In infants who require an $\mathrm{O}_{2}$ supplement, FRC can be measured in one of two ways: calibration is performed with gas volumes of the same $\mathrm{FN}_{2}$ as the infant is breathing, and FRC is calculated as described earlier; calibration is performed with room air, with the calculated volume subsequently being multiplied by a correction factor that accounts for the difference in $\mathrm{FN}_{2}$ between calibration and alveolar gas [3].

The bias flow $\mathrm{N}_{2}$ washout technique becomes inaccurate when the fractional concentration of inspired oxygen is $>0.7$ [3].

Helium $/ \mathrm{O}_{2}(80 / 20 \%)$ has been used in premature infants to prevent exposure to high $\mathrm{O}_{2}$ concentration [28] but may not be used interchangeably [29]. In addition, the effect of a helium/oxygen mixture on gas mixing, equilibration time and lung volume have yet to be evaluated [3]. While pure $\mathrm{O}_{2}$ has been suggested to decrease tidal breathing significantly $[30,31]$, there are no data indicating that any potentially harmful effects could result from such short exposures to pure $\mathrm{O}_{2}$ as during FRC measurement.

Data acquisition. Data acquisition requirements are dealt with elsewhere in this series [21, 26, 27, 32]. Particular points of relevance to lung volume measurements are: 1) a sampling rate of $100 \mathrm{~Hz}$ is normally adequate for the acquisition of $\mathrm{FRCN}_{2}$ data. However, $200 \mathrm{~Hz}$ may be required for rapidly breathing infants or the washout volume/time measurements (see later). 2) Prior to data acquisition, the operator should be prompted to enter whether a subject is being tested or an in vitro assessment performed, so that any body temperature, pressure and saturation (BTPS) corrections can be switched on or off, respectively. The type of measurement should be indicated in the report [11, 12]. 3) Prior to the FRC measurements, tidal flow and hence volume should be corrected to BTPS conditions, assuming that inspired air is at ambient temperature, pressure and saturation conditions (ATPS) and expired air at BTPS conditions [26]. 4) Volume drift, any drift of the tidal volume signal prior to switching the infant into $\mathrm{O}_{2}$ during FRC measurement should be minimized. 5) A representative end expiratory level must be established (see later). 6) In the absence of any injected air into the $\mathrm{O}_{2}$ circuit, there should be no drift in the baseline of the integrated nitrogen signal (INS), that is the INS should read zero (arbitrary units), after the three-way valve is activated. When testing patients with airway obstruction, the period during which there is no drift must persist for at least $90-120$ seconds $[11,12]$. 7) End of test should be operator-controlled rather than automatic. End of test is defined by a $\mathrm{FN}_{2}$ decreasing to 0.0065 within the mixing chamber and $\mathrm{N}_{2}$ analyser, in the presence of regular breathing. Before ending the washout, the operator must ensure that this low $\mathrm{N}_{2}$ concentration has been recorded 
during regular breathing. This can be confirmed by watching the movements of a collapsible breathing bag provided this has been incorporated in the washout circuit (see earlier) [11]. In the absence of such movements the test should continue, since the low $\mathrm{N}_{2}$ concentration may simply be due to a brief apnoea. The infant's head may need to be repositioned if there is any suggestion that such an apnoea could be due to a temporary upper airway obstruction rather than periodic breathing [11]. 8) For calculation of $\mathrm{FRCN}_{2}$ see later. Measured FRC must be converted to BTPS conditions.

Calibration of the nitrogen analyser. Equipment calibration has a significant influence on the calculated results and should be performed with utmost care and according to the manufacturer's recommendations. It is essential that: adequate equipment warming times be used according to the manufacturer's recommendation; calibration is performed with the same equipment configuration as during measurements; the calibration tools are checked periodically; qualified personnel, who understand both the procedure and data acquisition, perform the calibration; manual calibration is performed intermittently to check automatic calibration procedures.

After stabilization of the INS baseline, calibration with known low (LV) and a high (HV) room air volumes, below and above the infant's expected FRC, is performed. For a computerized system, integration of the mixed $\mathrm{FN}_{2}$ signal (INS) (arbitrary units) begins when the mixed $\mathrm{FN}_{2}$ value is $>0.006$. Washout is complete when $\mathrm{FN}_{2}$ falls $<0.0065$ in the mixing chamber [3].

The slope and intercept of the calibration line are calculated as follows [3]:

$$
\begin{aligned}
& \text { Slope }=\frac{(\mathrm{HV}-\mathrm{LV})}{(\mathrm{INS}(\mathrm{HV})-\mathrm{INS}(\mathrm{LV}))} \\
& \text { Intercept }=-(\mathrm{INS}(\mathrm{HV}) \times \text { Slope })+\mathrm{HV} \\
& \mathrm{FRC}=(\mathrm{INS} \times \text { Slope })+\text { Intercept }
\end{aligned}
$$

With any BTPS correction switched off, calibration should be confirmed by using the same syringe volume on two consecutive occasions; the calculated washout volume should be within $1 \%$ of the known volume. After infant testing has been completed, calibration should again be confirmed with a known volume after installing a clean $\mathrm{N}_{2}$ circuit to avoid contamination of the calibrating syringe. This is important to check that there has been no drift of the $\mathrm{N}_{2}$ analyser during the testing period. The use of calibration volumes equivalent to the infant's measured FRC is recommended, as is the use of similar $V \mathrm{~T} / \mathrm{FRC}$ ratios and respiratory rates to those of the infant $[7,9,11,12]$.

\section{Monitor display. See also FreY et al. [10].}

Tidal breathing prior to FRC assessment. During data collection and/or replay, time-based displays of flow and volume are required to observe breathing pattern and facilitate activation of the slide valve at end-expiration.

During measurement of $\mathrm{FRCN}_{2}$. Once the slide valve is activated, a time-based display of the following are required: the $\mathrm{N}_{2}$ concentration $\left(\mathrm{FN}_{2}\right)$ curve over time (s); the initial $\mathrm{N}_{2}$ concentration at the time of switching the subject into $\mathrm{O}_{2}$ (should read 0.00); the changes in the $\mathrm{N}_{2}$ concentration (displayed to two decimal points) during washout; the integrated $\mathrm{N}_{2}$ signal (arbitrary units).

After the washout is completed the operator needs to be able to: reject a curve before storage due to technical patterns, together with the ability to exclude (but not delete) selected trials during the analysis process; read off respective values of flow and volume during the tidal breathing recording prior to washout by, for example, moving a cursor through such data; examine/ print any cumulative calculations/plots of volume by washout as a function of time, e.g. from time zero up to a certain point in time or over any specified time period.

In summary, it is essential that both graphics and tabulation be of sufficiently high standard to allow the user to decide when a washout is complete. Furthermore, printouts of washout curves should be of sufficient resolution for later quality assurance. It is essential that such curves are saved for later inspection.

\section{Measurement protocol}

Further practical details of how to apply this technique and interpret results have been described previously. The task force has published a book that collates much of the relevant information and discusses background issues that may influence the measurement [3].

\section{Preparing the infant for measurements}

The following are necessary when preparing the infant for measurements: full resuscitation equipment, including suction, should be available at the site of infant lung function testing; two individuals (other than parents) should be present during testing, one of whom has the prime responsibility for the infant's well being. The infant must never be left unattended; the infant must be monitored continuously using at least a pulse oximeter; the hospital-specific protocol for sedation must be adhered to; measurements should be generally obtained with the sleeping infant laying supine. If other postures are used, these should be clearly indicated; the neck and/or shoulders should be supported in the midline in slight extension and position stabilized by using a neck-roll or head ring; the face mask should be transparent. It should cover the mouth and nose and be placed with minimal pressure. An airtight seal can be maintained with a thin ring of silicone putty; measurements should be restricted to periods of regular quiet breathing. It is particularly important to avoid switching the infant into $\mathrm{O}_{2}$ during rapid eye movement sleep when FRC may be very unstable [33]; if a squeeze jacket 
had been applied to perform forced expiatory manoeuvrers, it must be unfastened before measuring FRC.

\section{Preparation for collecting data for functional residual capacity}

The following is necessary when preparing for data collecting of the functional residual capacity: connecting the PNT-three way valve assembly to the face mask and recording at least $30 \mathrm{~s}$ of tidal breathing prior to FRC measurement; once a stable tidal breathing is observed on the monitor, switching the infant into $\mathrm{O}_{2}$ as close to end-expiration as possible; depending on the bias flow of $\mathrm{O}_{2}$ used and the length of the washout tube carrying the mixed $\mathrm{O}_{2} / \mathrm{N}_{2}$ gas from the infant to the $\mathrm{N}_{2}$ mixing chamber, the $\mathrm{FRCN}_{2}$ washout curve usually rises above the baseline within one $[11,12]$ or more seconds. It reaches an initial sharp peak followed by a stepwise decrease until the washout is complete. When the nitrogen analyser reads an $\mathrm{FN}_{2}$ of 0.0065 within the $\mathrm{N}_{2}$ mixing chamber at the end of the washout period, the three-way valve is activated switching the infant back into room air; the waiting period in between tests should be at least twice the washout time. After a washout has been completed and the infant is switched to room air, giving the infant a few sigh breaths via a Y-adapter carrying a bias flow $\left(12-15 \mathrm{~L} \cdot \mathrm{min}^{-1}\right)$ of air and connected to the PNT can speed the restoration of $\mathrm{FAi} \mathrm{N}_{2}[11]$.

\section{Potential sources of error when measuring $\mathrm{FRCN}_{2}$}

In addition to equipment related errors, factors that may adversely affect the accuracy of measurements [3, $7,9,11,12,25]$ include: insufficient equipment warming times; inadequate equilibration of the calibrating syringe with room air $\mathrm{N}_{2}$ after it had been used for calibration; mask or circuit leaks; drift of the tidal volume signal and changes in the end-expiratory level; baseline drifting of the $\mathrm{N}_{2}$ washout curve; PNT calibration errors and effect of heating; errors in estimating mask/apparatus dead space; retrograde movement of mixed $\mathrm{O}_{2}$ and $\mathrm{N}_{2}$ gas after it has passed beyond the $\mathrm{N}_{2}$ sampling needle port; switching errors above FRC; errors in BTPS correction; infant's apnoea during a washout; inadequate interval between washouts; malfunction of the gas sampling needle or the $\mathrm{N}_{2}$ analyser; incorrectly connecting the tube carrying the washout mixed $\mathrm{O}_{2} / \mathrm{N}_{2}$ gas (fig. 1) to the outlet instead of the inlet port of the $\mathrm{N}_{2}$ mixing chamber.

\section{Calculations}

Tidal breathing parameters. The tidal breathing parameters that need calculating include (see also BATEs et al. [26, 32]): End expiratory level (EEL); tidal volume; respiratory rate.

The end expiatory level (i.e. FRC) should be established over at least 5 breaths prior to switching the infant into $\mathrm{O}_{2}$, after correcting for any volume drift. For consistency, it is suggested that the EEL is calculated as the mean of all selected end expiratory points after drift correction, and that the selected level is clearly displayed on a time based trace for verification of accuracy by the operator. Some user flexibility may be required in those cases where EEL is particularly variable.

Calculation of $\mathrm{FRCN}_{2}$. As described earlier, the integrated mixed expired $\mathrm{FN}_{2}$ versus time $(t)$ is multiplied by the constant flow of $\mathrm{O}_{2}\left(V^{\prime}\right)$ to obtain the volume of expired $\mathrm{N}_{2}\left(V \mathrm{~N}_{2}\right)$ :

$$
V \mathrm{~N}_{2}(t)=V^{\prime} \int_{0}^{\mathrm{t}} \mathrm{FN}_{2}(t) d t
$$

The total volume ( $V$ tot) measured by the washout, which is at ATPS, is calculated (see earlier):

$$
V \text { tot }=V_{\mathrm{N}_{2}} \div\left(\mathrm{FAi}_{\mathrm{N}_{2}}-0.02\right)
$$

The volume of apparatus dead space $(V \mathrm{ds}$,app $(\mathrm{mL}))$, including the face mask (see earlier), (assumed to be at ATPS) is subtracted from $V$ tot to obtain the effective washout volume ( $V$ eff):

$$
V \text { tot }-V_{\text {ds,app }}=V_{\text {eff }}
$$

The $V$ eff is converted to BTPS ( $V$ eff-BTPS) [24].

The switching errors above FRC should be corrected for by subtracting any volume above EEL (previously converted to BTPS conditions) from the VEFF-BTPS to obtain $\mathrm{FRCN}_{2}$ [7, 12]:

$$
\mathrm{FRCN}_{2}=V \text { eff- }-B T P S-V>\text { FRC-BTPS }
$$

Switching errors should be negligible if automatic switching into $\mathrm{O}_{2}$ has been employed and the infant has regular breathing (see earlier).

FRC should be calculated from at least two technically acceptable measurements that are within $10 \%$ or $10 \mathrm{~mL}$ of each other, whichever is the larger or, in the presence of increased variability, the mean of three technically satisfactory trials.

Quality control parameters. $\mathrm{FRCN}_{2}$ is the only "outcome measure" that will be routinely reported from the nitrogen washout technique. Calibration and raw data of FRC should be saved in ASCII or similar format. Data that should be retrievable for validation purposes by the user, but not necessarily visible, include the uncalibrated analogue-to-digital (A/D) signal and all intermediate calculations leading to the final results. The parameters that should be displayed/available for each individual trial to assist in user or automated selection of the "best" data and to provide overall quality assurance, are listed below. Since many of these quality control features need to be summarized in publications describing $\mathrm{FRCN}_{2}$ measurements in infants, it is essential that such information can be automatically saved and, if required, exported to a suitable spreadsheet.

The use of standard abbreviations as indicated would be of considerable benefit and is strongly recommended. The quality control and other parameters include: number of acceptable measurements (n); total number of measurements performed (n-tot); volume of apparatus dead space $(V \mathrm{ds}, \mathrm{app})(\mathrm{mL})$; type and size of mask e.g. Rendall Baker size; mean tidal volume prior to FRC measurement $(V \mathrm{~T}(\mathrm{~mL}))$; mean 
respiratory rate prior to FRC measurement (RR $\left(\min ^{-1}\right)$ ); minute ventilation (expired minute ventilation) prior to FRC measurement, calculated as $\mathrm{RR} \times V \mathrm{~T}\left(V^{\prime} E\right.$; also referred to as MV); stability of EEL prior to switching the infant into $\mathrm{O}_{2}$, expressed as $\%$ variability of end expiratory points over "n" (five) breaths prior to each switching of the infant into $\mathrm{O}_{2}$ during FRC measurement (EEL\%) [26, 27]; stability of EEL prior to each switching into $\mathrm{O}_{2}$ during FRC measurement, expressed as the SD of the end expiratory points relative to the baseline over " $n$ " (five) breaths prior to each manoeuvre (EEL-s, (mL)) [26, 27]; volume above EEL reflecting the switching error above FRC $(V>$ FRC $(\mathrm{mL}))$; FRC washout time $\left(t \mathrm{FRCN}_{2}(\mathrm{~s})\right)$; cumulative volume washed out at 50,75 , and $85 \%$ of the (total) washout time, FRC $50 \mathrm{~N}_{2}$, FRC75N 2, FRC $85 \mathrm{~N}_{2}$ (the potential usefulness of such parameters have yet to be explored).

\section{Reporting}

Data should be reported according to the following: for FRC $(\mathrm{mL})$ the mean from three trials or a minimum of two if these are within $10 \%$ or $10 \mathrm{~mL}$ of each other (see earlier); the coefficient of variation $(\mathrm{CV}=100 \times \mathrm{sD} / \mathrm{mean})$ of $3-5$ technically satisfactory FRC measurements should be available as a measure of the intra-subject variability; for quality control, a print out of the $\mathrm{FRCN}_{2}$ nitrogen washout curve should also be provided; a print out of the time-based flow and volume traces at the time of switching into $\mathrm{O}_{2}$ is helpful; ideally, data should not be presented as "per cent of predicted"; predicted values of $\mathrm{FRCN}_{2}$ beyond the neonatal period should be expressed as the regression of FRC on crown-heel length along with the residual standard deviation (RSD). Observed values of $\mathrm{FRCN}_{2}$ can then be reported as the number of RSD removed from the predicted mean [34]; $\mathrm{FRCN}_{2}$ may be expressed per unit body weight during the first month of life as the regression of FRC versus weight is fairly linear and passes close to zero. $\mathrm{FRCN}_{2}$ should never be expressed per unit body length, as neither of the conditions discussed earlier are met [34]; results can also be expressed in relation to the "normal range" according to the infant's age and sex; a reference equation from collated data of FRCHe [34] (table 1) that can be used until sufficient reference data is obtained for $\mathrm{FRCN}_{2}$ is:

$$
\mathrm{FRCHe}=0.0036 \cdot \mathrm{L}^{2.531}
$$

where $\mathrm{L}$ is the crown-to-heel length in $\mathrm{cm}, \mathrm{RSD}=17.7 \%$ and $95 \%$ confidence interval $(\mathrm{CI})=71-141 \%$.

See also Tepper et al. [3]; Stocks and Quanjer [34].

Table 1.-Representative values of functional residual capacity at arbitrary chosen values of length and body weight in infants from collated data [34]

\begin{tabular}{lccccc}
\hline Length cm & 50 & 60 & 70 & 80 & 90 \\
FRCHe mL & 72 & 114 & 168 & 236 & 318 \\
$95 \% \mathrm{CI} \mathrm{mL}$ & $51-102$ & $81-161$ & $119-237$ & $166-333$ & $226-448$ \\
\hline
\end{tabular}

FRCHe: Functional residual capacity by helium; CI: confidence interval.
Each individual laboratory should attempt to study at least some healthy infants to check whether available reference data are appropriate for their population. Regular checks should also be made to ensure that the equipment produces appropriate values during in vitro assessment over the full range of lung volumes likely to be encountered during studies.

\footnotetext{
Acknowledgements. The authors would like to thank all the other members of the Task Force who contributed to developing these recommendations: J. Allan (Philadelphia, PA, USA), E. Bar-Yishay (Jerusalem,Israel), J.H.T. Bates (Montreal, Canada), C. Beardsmore (Leicester, UK), R. Castile (Columbus, OH, USA), J.B. Clough (Southampton, UK), A.L. Coates (Toronto, Canada), I. Dundas (London, UK), D. Filbrun (Colombus, OH, USA), U. Frey (Berne, Switzerland), S. Godfrey (Jerusalem, Israel), R. Gregson (Southampton, UK), M. Henschen (Freiburg, Germany), A-F Hoo (London, UK), A. Jackson (Boston, MA, USA), J. de Jongste (Rotterdam, the Netherlands), R. Kraemer (Berne, Switzerland), S. Lum (London, UK), P. Merkus (Rotterdam, the Netherlands), I.T. Merth (Leiden, the Netherlands), B. Reinmann (Berne, Switzerland), G. Schmalisch (Berlin, Germany), P. Seddon (Brighton, UK), G. Sharma (Chicago, IL, USA), M. Silverman (Leicester, UK), P.D. Sly (West Perth, Western Australia), D. Vilozni (Sharon, Israel), E. van der Wiel (Rotterdam, the Netherlands) and to members of the industry who read the various drafts and provided invaluable feedback.
}

\section{References}

1. American Thoracic Society/European Respiratory Society. Respiratory mechanics in infants: physiological evaluation in health and disease. Am Rev Respir Dis 1993; 147: $474-496$.

2. Gaultier C. Lung volume in neonates and infants. Eur Respir J 1989; 2: Suppl 4, 130s-134s.

3. Tepper RS, Merth IT, Newth CJL, Gerhart T. Measurement of functional residual capacity in infants by helium dilution and nitrogen washout techniques. In: Stocks J, Sly PD, Tepper RS, Morgan WJ, eds. Infant Respiratory Function Testing. New York, John Wiley \& Sons, Inc., 1996; pp. $165-189$.

4. Sjöqvist BA, Sandberg K, Hjalmarson O, Olsson T. Calculation of lung volume in newborn infants by means of a computer-assisted nitrogen washout method. Pediatr Res 1984; 18: 1160 - 1164.

5. Gerhardt T, Hehre D, Bancalari E, Watson H. A simple method for measuring functional residual capacity by nitrogen washout in animals and newborn infants. Pediatr Res 1986; 20: 668-671.

6. Sivan Y, Deakers TW, Newth CJL. An automated bedside method for measuring functional residual capacity by nitrogen washout in mechanically ventilated children. Pediatr Res 1990; 28: 446-450.

7. Gappa M, Fletcher ME, Dezateux CA, Stocks J. Comparison of nitrogen washout and plethysmographic measurements of lung volume in healthy infants. Am Rev Respir Dis 1993; 148: 1496-1501. 
8. Merth IT, Verschragen GJ, Olievier ICW, de Winter JP, Quanjer $\mathrm{PhH}$. Watersealed spirometer for measurements in newborns and infants. $J$ Appl Physiol 1993; 74: 470-475.

9. Gustafsson PM, Johansson HJ, Dahlbäck GO. A pneumotachometric nitrogen washout method for measurement of the volume of trapped gas in the lungs. Pediatr Pulmonol 1994; 17: 258 -268.

10. Newth CJL, Enright P, Johnson RL. Multiple-breath nitrogen washout techniques: including measurements with patients on ventilators. Eur Resp J 1997; 10: $2174-2185$.

11. Morris MG. The open circuit nitrogen washout technique for measuring the lung volume in infants: Methodological Aspects. Thorax 1999; 54: 790-795.

12. Morris MG. A novel noninvasive technique for measuring the residual lung volume by nitrogen washout with rapid thoracoabdominal compression in infants. Thorax 1999; 54: 874-883.

13. Brown R, Leith DE, Enright PL. Multiple breath helium dilution measurement of lung volumes in adults. Eur Respir J 1998; 11: 246-255.

14. Schulze A, Schaller P, Topfer A, Kirpalani H. Measurement of functional residual capacity by sulfur hexafluoride in small-volume lungs during spontaneous breathing and mechanical ventilation. Pediatr Res 1994; 35: 494-499.

15. Hammer J, Newth CJL. Infant lung function testing in the intensive care unit. Intensive Care Med 1995; 21: $744-752$.

16. Hammer J, Numa A, Newth CJ. Total lung capacity by $\mathrm{N}_{2}$ washout from high and low lung volumes in ventilated infants and children. Am J Respir Crit Care Med 1998; 158: 526-531.

17. Numa AH, Hammer J, Newth CJL. Effect of prone and supine positions on functional residual capacity, oxygenation, and respiratory mechanics in ventilated infants and children. Am J Respir Crit Care Med 1997; 156: $1185-1189$.

18. Shao H, Sandberg K, Sjöqvist B, Hjalmarson O. Moment analysis of multibreath nitrogen washout in healthy preterm infants. Pediatr Pulmonol 1998; 25: $52-58$.

19. Wauer RR, Maurer T, Nowotny T, Schmalisch G. Assessment of functional residual capacity using nitrogen washout and plethysmographic techniques in infants with and without bronchopulmonary dysplasia. Intensive Care Med 1998; 24: 469-475.

20. Frey U, Stocks J, Coates A, Sly P, Bates J. Specifications for equipment used for infant pulmonary function testing. Eur Resp $J$ 2000; 16: $731-740$.

21. Frey U, Stocks J, Sly P, Bates J. Specifications for signal processing and data handling used for infant pulmonary function testing. Eur Resp $J$ 2000; 16: $1016-1022$.

22. Lundin G. Nitrogen elimination during oxygen breathing. Acta Physiol Scand 1953; 30: 130-143.

23. Groom AC, Morin R, Fahri LE. Determination of dissolved nitrogen in blood and investigation of nitrogen washout from the body. $J$ Appl Physiol 1967; 23: 706-712.

24. Robertson JS, Siri WE, Jones HB. Lung ventilation patterns determined by analysis of nitrogen elimination rates: use of mass spectrometer as a continuous gas analyzer. J Clin Invest 1950; 29: $577-590$.

25. Morris MG. A simple new technique to measure the effective dead space of the face mask with a water volumeter in infants. Eur Respir J 1999; 14: 1163 1166.

26. Bates J, Schmalisch G, Filbrun D, Stocks J. Tidal breath analysis for infant pulmonary function testing. Eur Respir J 2000; 16: 1180-1192.

27. Sly PD, Tepper RS, Henschen M, Gappa M, Stocks J. Standards for infant respiratory function testing: Tidal forced expirations. Eur Resp J 2000; 16: 741 - 748.

28. Poets CF, Rau GA, Neuber K, Gappa M, Seidenberg J. Determinants of lung volume in spontaneously breathing preterm infants. Am J Resp Crit Care Med 1997; 155: 649-653.

29. Gappa M, Schulz KN, Poets CF, von der Hardt H. Comparison of heliox and oxygen for measurement of FRC in infants with bronchopulmonary dysplasia (BPD). Eur Respir J 1997; 10: 166s.

30. Gustafsson PM, Källman S, Bhiladvala M, Fletcher ME. $\mathrm{N}_{2}$-washout with pure oxygen reduces tidal volumes markedly in sedated infants. Eur Respir $J$ 1997; 10 Suppl. 25, 165s.

31. Schibler A, Schneider M, Frey U, Kraemer R. Moment ratio analysis of multiple breath nitrogen washout in infants with lung disease. Eur Respir $J$ 2000; 15: 1094-1101.

32. Bates JHT, Turner MJ, Lanteri CJ, Jonson B, Sly P. Measurement of flow and volume. In: Stocks J, Sly PD, Tepper RS, Morgan WJ, eds. Infant Respiratory Function Testing. New York, John Wiley \& Sons, Inc., 1996; pp. 81-116.

33. Gaultier C, Fletcher M, Beardsmore C, Motoyama E, Stocks J. Measurement conditions. In: Stocks J, Sly PD, Tepper RS, Morgan WJ, eds. Infant Respiratory Function Testing. New York, John Wiley \& Sons, Inc., 1996; pp. 29-44.

34. Stocks J, Quanjer PhH. Reference values for residual volume, functional residual capacity and total lung capacity. Eur Respir J 1995; 8: $492-506$. 